\title{
Vacancy Patterning and Patterning Vacancies: Controlled Self-Assembly of Fullerenes on Metal Surfaces
}

\author{
Alexander Kaiser ${ }^{\$}$, Francesc Viñes $\$$, Francesc Illas ${ }^{\$ *}$, Marcel Ritter ${ }^{*}$, Frank Hagelberg ${ }^{\#}$, Michael \\ Probsts \\ Institut für Ionenphysik und Angewandte Physik, University of Innsbruck, Technikerstrasse 25, \\ A-6020 Innsbruck, Austria \\ separtament de Química Física \& Institut de Química Teòrica i Computacional \\ (IQTCUB), Universitat de Barcelona, c/Martí i Franquès 1, E-08028 Barcelona, Spain \\ "Institute for Basic Sciences in Engineering Science, University of Innsbruck, 6020, \\ Technikerstraße 13, Austria \\ \#Department of Physics and Astronomy, East Tennessee State University, Johnson City, TN \\ 37614, USA \\ e-mails: Michael.Probst@uibk.ac.at; francesc.illas@ub.edu
}

\begin{abstract}
A density functional theory study accounting for van der Waals interactions reveals the potential of metal surface vacancies as anchor points for the construction of user-defined 2D patterns of adsorbate molecules via a controlled self-assembly process. Vice versa, energetic criteria indicate the formation of regular adsorbate-induced vacancies after adsorbate self-assembly on clean surfaces. These processes are exemplified by adsorbing $\mathrm{C}_{60}$ fullerene on $\mathrm{Al}(111), \mathrm{Au}(111)$, and $\mathrm{Be}(0001)$ surfaces with and without single, triple, and septuple atom pits. An analysis of vacancy-adatom formation energetics precedes the study of the adsorption processes.
\end{abstract}

Keywords: Adsorption $\cdot$ Self-Assembly $\cdot$ Fullerene $\cdot$ Vacancy Formation $\cdot$ Nanopatterns $\cdot$ Metal Surfaces 


\section{Introduction}

The increasing research interest on Self-Assembled Monolayers (SAMs), patterned arrangements typically consisting of organic molecules, is related to their promising applications in biosensors, ${ }^{1}$ optoelectronics, ${ }^{2}$ and tribology. ${ }^{3}$ Typically, such arrangements are studied on metal surfaces, although recent research endeavors have been applied on SAM formation on graphene layers, an appealing field for graphene bandgap engineering, ${ }_{4}^{4}$ but also in order to get, for instance, ordered magnetic organic layers. On the other hand defined vacancy patterns on surfaces were created, on a nanometer scale, as early as in 2002 within the millipede project.$^{5}$ It involved a two-dimensional (2D) array of Atomic Force Microscopy (AFM) tips which resembled nm-scale millipedes which imprinted user-defined patterns of vacancies on a given thin polymer surface.

Surface vacancies are particularly attractive adsorption sites for subsequently deposited molecules, given the low-coordination of vacancy neighboring surface atoms, and their concomitant improved chemical activity. When the adsorption of a molecule turns out to be thus strongly enhanced by the presence of a vacancy, as compared to the clean surface, individual population of vacancies can become preferred to molecular clustering, i.e. SAM formation. Thus, a vacancy-determined pattern of adsorbate molecules can be constructed. We call this process Controlled Self-Assembly (CSA), and we shall investigate below its feasibility taking fullerene $\mathrm{C}_{60}$ adsorption on gold, aluminum, and beryllium surfaces as cases and key examples.

Another related process is a type of SAM formation which, on the contrary, induces the creation of surface vacancies. Conceptually, the fullerenes, first adsorbed on the clean surface, would induce vacancy formation beneath them and reside in their self-made 'nests'. Vacancy formation triggered by the adsorption of a molecule or a cluster on a surface is not rare, and has been observed, for instance, on $\mathrm{Pt}$ clusters on $\mathrm{CeO}_{2}$ substrates. As far as fullerenes are considered, vacancy incitement in the course of $\mathrm{C}_{60}$ monolayer formation on various surfaces has been investigated by many experimental and theoretical studies. $\mid$ On one hand, these structures remain stable at Room Temperature $(R T),{ }^{7 \mathrm{~d}}$ and $\mathrm{C}_{60}$ adsorption is found to induce dimple formation on the $\mathrm{Au}(111)$ substrate, with well-separated individual $\mathrm{C}_{60}$ nucleation centers for cluster formation upon continued deposition. Vacancies were found to compete with step edges for adsorbates, underlying the importance of under-coordination in the increased adsorbent capacity. On the other hand Tzeng et al. observed that annealing to $750 \mathrm{~K}$ desorbs all $\mathrm{C}_{60}$ molecules of $\mathrm{Au}(111)$ while preserving the structure of $\mathrm{C}_{60} \cdot{ }^{8}$ However, vacancy formation was not discussed in their work. ${ }^{8}$
Fransis 12/4/2014 12:05

Comentario [1]: Add Ref. Nature Physics 2013, 9,368 [This is now correct].

Fransis 12/4/2014 12:06

Comentario [2]: We agreed on (i) put a single number per reference and (ii) avoid overcitation, i.e. for giving a feed-back that there is much research, one can cite a few cases only. 
The adsorption of $\mathrm{C}_{60}$ on metal surfaces is indeed a topic that has driven much attention over the last years, and the nature of the $\mathrm{C}_{60}$ substrate chemical bonding is still a matter of

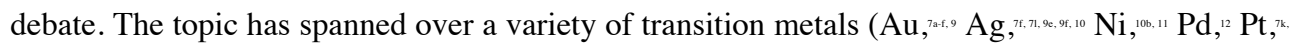

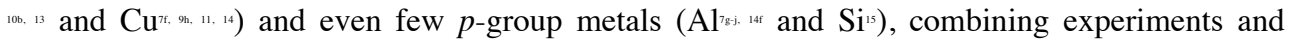
theoretical calculations. The latter mostly rely on Density Functional Theory (DFT), although for such systems the inclusion of a proper description of van der Waals $(v d W)$ dispersive forcers may be determinant. Two recent studies of $\mathrm{C}_{60}$ adsorbed on smooth $\mathrm{Au}(111)$ and $\operatorname{Ag}(111)$ surfaces with and without van-der-Waals (vdW) correction revealed a strong covalent, partly ionic interaction of $\sim 1-3 \mathrm{eV}$ strength, where the metal $\mathrm{C}_{60}$ charge transfer plays a key role. ${ }^{\text {gb, ge }}$ However, pristine metal surfaces are found to reconstruct when exposed to fullerenes: Note that a clean $\mathrm{Au}(111)$ surface tends to adopt a herringbone-structure, ${ }^{16}$ which, as revealed by X-Ray Diffraction $(X R D)$ measurements and structural analysis, undergoes at $160{ }^{\circ} \mathrm{C}$ a surface transformation accompanied by removal of the herringbone structure and introduction of surface vacancies. Fullerenes were found to reside atop of a regular $(2 \sqrt{3} \times 2 \sqrt{ } 3)$ R $30^{\circ}$ pattern of vacancies with $\mathrm{C}$-Au distances of $2.49 \AA^{7 \mathrm{~b}}{ }^{\mathrm{b}}$ These surface reconstructions, such as vacancy or crater formation, were experimentally observed and confirmed by DFT theory for $\mathrm{C}_{60}$ adsorbed on $\operatorname{Pt}(111),{ }^{7 \mathrm{k}} \mathrm{Ag}(111),{ }^{71} \mathrm{Al}(111),{ }^{7 \mathrm{i}, 7 \mathrm{j}}$ and $\mathrm{Au}(110) .^{7 \mathrm{e}}$

Here we consider representatives and structurally similar surfaces of a noble metal, $\mathrm{Au}(111)$, a $p$-group metal, $\mathrm{Al}(111)$, and an alkaline earth metal, $\mathrm{Be}(001)$. These metals were selected for several different reasons; most of the related literature deals with $\mathrm{C}_{60}$ adsorption on $\mathrm{Au}$, which therefore is presented as a good reference system and test field. Secondly, Al is used as electrode material in organic photovoltaics, where it is in contact with the functionalized fullerene Phenyl- $\mathrm{C}_{61}$-Butyric acid Methyl ester $(P C B M) .{ }^{17}$ Lastly, Be is a metal candidate for walls of future fusion reactors, ${ }^{18}$ and studies of the interaction of carbonaceous materials with beryllium are, to the best of our knowledge, very rare. Experiments with Be need special precautions due to its high toxicity. Therefore it is interesting to now how elements adjacent to it in the periodic table could serve as proxies for Be. Comparing the energetics of $\mathrm{Be}$ and $\mathrm{Al}$ in this work also serves this purpose.

At variance with most of the large amount of work already done in the field, we considered well-separated $\mathrm{C}_{60}$ molecules in large unit cells to specifically assess the interaction of $\mathrm{C}_{60}$ with the surface, contrary to previous studies dealing with close-packed $\mathrm{C}_{60}$ monolayers. 
Furthermore, our approach explicitly includes vdW forces, which has only been accounted for in one publication of $\mathrm{C}_{60}$ adsorption on $\mathrm{Au}(111)$ where, however, vacancy formation was not considered. $^{9 \mathrm{~b}}$ We also analyze vacancy adatom formation energetics by comparing adsorption energies, charge transfer, and bond nature for $\mathrm{C}_{60}$ adsorption on clean surfaces, as well as in single, triple, and septuple atom pits. Energetic criteria for vacancy formation inducement and controlled self-assembly are discussed.

\section{Computational Details}

All DFT calculations and structural optimizations were carried out using the VASP code. ${ }^{19}$ A basis set of Plane-Waves $(P W)$ was used with a PW kinetic energy cutoff of $415 \mathrm{eV}$. Valence electrons were described using the Perdew-Burke-Ernzerhof (PBE) exchangecorrelation functional, whereas core electrons were described by using the Projector Augmented Wave $(P A W)$ method. The vdW forces are treated by the long-range correction as proposed by Grimme. ${ }^{19 a, 20}$ Default vdW parameter values were used for $\mathrm{C}, \mathrm{Al}$, and $\mathrm{Be}$ atoms, whereas for $\mathrm{Au}$ atoms we used the values supplied by Amft et al. ${ }^{21}$ A sufficiently accurate k-point mesh was chosen for all calculations, which happened to be the $\Gamma$ point for large cells

A $(5 \times 5)$ unit cell was chosen for the six atomic layer slab model representing the $\mathrm{Al}(111)$ and $\mathrm{Au}(111)$ surfaces with two frozen and four flexible layers -i.e. $(2+4)$ approximation - and a vacuum thickness of $14 \AA$, similar to that used previously in related works. ${ }^{9 e}$ A larger $(8 \times 8)$ unit cell had to be used for the $\mathrm{Be}(0001)$ surface, in order to achieve a large enough separation of $\mathrm{C}_{60}$ from its periodic images. The distance between the carbon atoms of $\mathrm{C}_{60}$ and their periodic images is in all simulations larger than $7 \AA$, giving results close to vacuum conditions. In the direction perpendicular to the surface, the distance between the topmost carbon atoms and the bottom metal layer of the periodic slab image is always larger than $6.5 \AA$. To construct input geometries, $\mathrm{C}_{60}$ was manually located above the surface or the center of a vacancy with one of its carbon hexagons pointing downwards at a substantially larger distance than that found in the optimized geometry. During the optimization $\mathrm{C}_{60}$ is allowed to fully relax, as well as the top four surface metal layers.

We first validated our computational settings by optimizing metal lattice constants $(a$, in case of Be also $c$ ) and calculating cohesive energies $(C E)$, work functions $(W F)$ and surface energies $(S E)$. As can be seen in Table 1 we found good agreement with literature (lit.) values. 
Our resulting optimized lattice constants were adopted throughout this work. Last but not least, molecules were visualized in the Vish visualization shell ${ }^{31}$. An estimated number of 44 single core wall clock years on Intel Xeon E5 and similar processors was spent in total for the optimizations.

Table 1: Lattice constants ( $a, c$ in $\AA$ ), cohesive energies (CE in $\mathrm{eV}$ ), work functions (WF in $\mathrm{eV}$ ), and surface energies ( $\mathrm{SE}$ in $\mathrm{J} / \mathrm{m}^{-2}$ ) compared with literature data.

\begin{tabular}{ccccccccc}
\hline & $\boldsymbol{a} / \boldsymbol{c}$ & lit. & $\boldsymbol{C E}$ & lit. & $\boldsymbol{W F}$ & lit. & $\boldsymbol{S E}$ & lit. \\
\hline \hline Al & 4.013 & $4.04^{22}$ & 3.73 & $3.23-4.21^{23}$ & 4.13 & $4.24-4.54^{24}$ & 1.1 & $0.94-1.27^{24 \mathrm{~b}, 25}$ \\
Au & 4.082 & $4.079^{22}$ & 3.74 & $3.73-3.97^{26}$ & 5.06 & $4.83-6.01^{24 \mathrm{~b}, 27}$ & 1.4 & $1.25-1.61^{24 \mathrm{~b}, 28}$ \\
Be & $2.236 /$ & $2.282^{29} /$ & 3.85 & $3.32-4.0^{30}$ & 5.27 & $4.98-5.62^{24 \mathrm{~b}}$ & 2.1 & $2.1-2.7^{24 \mathrm{~b}}$ \\
& 3.54 & $3.575^{29}$ & & & & & & \\
\hline
\end{tabular}

\section{Vacancy Formation}

First we dealt with the formation of surface vacancies, since they, through surface reconstruction, can enhance the binding strength of adsorbed $\mathrm{C}_{60}$ and even lower the total energy of the $\mathrm{C}_{\mathrm{o}} /$ surface system. Often these vacancies are termed pits or craters. Deep pits with 3 or 7 atom vacancies have shown to increase adsorption energies of the $\mathrm{C}_{60} / \mathrm{Au}(111)$ system according to DFT calculations without dispersion corrections. ${ }^{\text {‘d }}$ Single-atom and regular multi-atom vacancies have been optimized starting from pristine ideal surfaces, from which 1,3 , or $7(v)$ atoms have been removed. Three-atom vacancies form a triangular pit, whereas seven-atom vacancies form a hexagonal pit. These structures are similar to those depicted by Tang et al., yet residing in a larger unit cell..$^{7 d}$

The vacancy-adatom formation energy is defined $\operatorname{as}^{32}$

$$
E_{\mathrm{va}}(v)=T(-v)+T(v)-2 T(0),
$$

where $T( \pm v)$ is the total energy of the clean surface, $T(0)$, with $v$ additional or $-v$ removed atoms, i.e. $T(-3)$ is the total energy of a surface with a three-atom vacancy, whereas $T(7)$ is the total energy of a surface with seven adatoms. $E_{v a}$ is defined as the energetic cost of building a vacancy from a clean surface, where the removed atoms attach, as a cluster, to the surface at an infinite distance from the vacancy. Thus the vacancy-adatom formation energy $E_{v a}$ can be represented as sum of the adatom formation energy, $E_{a d}$, and the vacancy formation energy, $E_{\text {vac }}$. Both are defined with reference to the total energy of a bulk atom, $T_{\text {bul }}$ : 


$$
\begin{aligned}
& E_{a d}(v)=T(v)-T(0)-v T_{\text {bulk. }} \\
& E_{\text {vac }}(v)=T(-v)+v T_{\text {bulk }}-T(0) .
\end{aligned}
$$

In other words, $E_{a d}$ is the energy required to remove $v$ atoms from the bulk and add them collectively on top of the surface, whereas $E_{v a c}$ is the energy gained by removing $v$ atoms from the clean surface and adding them to the bulk reservoir. ${ }^{33}$ The adatom binding energy $E_{b a d}$ and the vacancy binding energy $E_{\text {buc }}$ are calculated using the atomic vacuum energy, $T_{a}$, as reference energy instead of the bulk value;

$$
\begin{aligned}
& E_{\text {bad }}(v)=T(0)+v T_{a t}-T(v), \\
& E_{\text {prac }}(v)=T(-v)+v T_{a t}-T(0) .
\end{aligned}
$$

Thus the energy $E_{\text {bad }} / E_{\text {brac }}$ is that needed to remove one by one the ad/vacancy atoms from the surface to the vacuum. The adiabatic removal of an atom from the surface can be seen as a two-step process, where vacancy-adatom formation is followed by removal of the adatom. Note that the overall five defined energies are not independent from each other:

$$
\begin{aligned}
& E_{v a}=E_{a d}+E_{v a c}, \\
& E_{v a}+E_{b a d}=E_{b r a c} .
\end{aligned}
$$

The energetic results for each surface and vacancy size are summarized in Table 2 together with the structural deformation $d_{\mathrm{c}}$. The deformation $d_{f f}$ is measured as the distance of an atom to its initially ideal crystal position in percent of the nearest neighbor distance and corrected for periodic boundary conditions, and Table 2 shows the maximal values.

From our findings, $E_{v a}$ is the most important value for the prediction of possible surface reconstruction, since it is the minimum energy that has to be compensated by the interaction between the adsorbate species $\left(\mathrm{C}_{60}\right.$ in our case) and the surface to allow for vacancy formation. In particular, the dissociation energy $D$ for removing $\mathrm{C}_{60}$ from the surface vacancy - also called the adsorption energy - needs to be even larger than the sum of $E_{s a}$ and $D$ for the case of a clean surface so as to make the reconstruction energetically favorable. From the $E_{v a}$ values of Table 2 we observe that, for a single atom vacancy, $\mathrm{Be}(2.09 \mathrm{eV})$ is more stable than $\mathrm{Al}(1.6 \mathrm{eV})$, which is in turn more stable than $\mathrm{Au}(1.11 \mathrm{eV})$. The enhanced stability of Be could be explained by its higher melting point $(1551 \mathrm{~K})$ compared to $\mathrm{Al}(933 \mathrm{~K})$, and $\mathrm{Au}(1338 \mathrm{~K})$. $^{34}$ However, by this line of reasoning, Au should be more stable than Al, which is not reflected by our results. In contrast, they suggest that surface reconstruction should proceed more easily in gold than in aluminum or beryllium. $E_{v a}$ is, as expected, not directly proportional to $v$, as fewer bonds-to-neighbors have to 
be broken for the removal of more and more atoms. An increase by a factor of 1.8-2.2 is observed when going from one- to three-atom vacancies, and of 2.3-3.2 from one- to seven-atom vacancies. Thus, after the first three atoms removed, removing any additional atom comes at a significantly reduced energy expense ( circa 147, 258, and $278 \mathrm{meV}^{\text {atom }}{ }^{-1}$ for $\mathrm{Al}, \mathrm{Au}$, and $\mathrm{Be}$, respectively). It should be noted that in the case of septuple vacancy pits the $5 \times 5 \times\left(6+\mathrm{L}_{z}\right)$ the unitcell might be too small and interactions between neighboring pits could influence our results, since only two rows of atoms separate the craters. In these cases results should be taken as mere indications rather than absolute values.

Table 2: Energies of vacancy-, adatom-, and vacancy-adatom formation as well as of structural deformation for single, triple, and septuple atom pits.

\begin{tabular}{|c|c|c|c|c|c|c|c|}
\hline & $v$ & $E_{n} / \mathbf{e V}$ & $E_{a a} / \mathbf{e V}$ & $\boldsymbol{E}_{\mathrm{ral}} / \mathbf{e V}$ & $\boldsymbol{E}_{b a d} / \mathrm{eV}$ & $E_{b \text { bud }} / \mathbf{e V}$ & $d_{f f} / \%$ \\
\hline \multirow[t]{3}{*}{ Al } & 1 & 1.60 & 0.91 & 0.69 & 2.83 & 4.42 & 18.7 \\
\hline & 3 & 3.11 & 1.59 & 1.52 & 9.61 & 12.72 & 52.3 \\
\hline & 7 & 3.70 & 2.23 & 1.48 & 23.91 & 27.61 & 70.5 \\
\hline \multirow[t]{3}{*}{ Au } & 1 & 1.11 & 0.53 & 0.58 & 3.20 & 4.31 & 3.0 \\
\hline & 3 & 2.49 & 1.30 & 1.20 & 9.91 & 12.40 & 9.6 \\
\hline & 7 & 3.52 & 1.76 & 1.76 & 24.39 & 27.91 & 7.5 \\
\hline \multirow[t]{3}{*}{$\mathbf{B e}$} & 1 & 2.09 & 0.84 & 1.25 & 3.01 & 5.11 & 7.1 \\
\hline & 3 & 3.77 & 1.40 & 2.36 & 10.16 & 13.92 & 8.0 \\
\hline & 7 & 4.88 & 2.13 & 2.74 & 24.84 & 29.72 & 12.8 \\
\hline
\end{tabular}

A deep inspection of the formation energy values encompassed in Table 2 unveils that the two contributions to $E_{\text {ra }}\left(E_{a d}\right.$ and $\left.E_{v a}\right)$ carry almost equal weights for $\mathrm{Au}$. In the case of $\mathrm{Al}$ the adatom formation energy dominates, whereas in case of Be it is the vacancy formation energy. 
Regardless of the case, $E_{b a d}$ and $E_{b \text { bra }}$ scale with $v$. Single-atom vacancy binding energies $E_{b \text { bac }}$ are larger than cohesive energies for the three metals. This means that the removal of a single atom from the surface takes more energy than removing an atom from the perfect bulk. Thus, indirectly, $E_{\text {bac }}$ carries information about the stability of a material against sputtering, in a better fashion than the cohesive energy. As far as deformation is concerned, Al deforms rather dramatically whereas $\mathrm{Au}$ and $\mathrm{Be}$ vary much less. Overall the deformation increases from the fixed bottom layers to the top layer and towards the vacancies or adatoms.

The computed vacancy and adatom formation energies appear to be in accord to previous computed values in the literature. In particular, the $E_{v a}$ formation energy of $1.72 \mathrm{eV}$ reported by Stumpf and Scheffler for $\mathrm{Al}(111)^{33}$ is composed of $E_{\text {vac }}=0.67 \mathrm{eV}$ and $E_{a d}=1.05 \mathrm{eV}$. Less agreement is found with DFT calculations within the Local Density Approximation (LDA) which yielded an $E_{v a c}$ energy of $0.36 \mathrm{eV}$ for $\mathrm{Al}(111),{ }^{35}$ and $0.83 \mathrm{eV}$ for $\mathrm{Au}(111){ }^{r}$ The value for $\mathrm{Al}(111)$ seems especially low since another study within the Generalized Gradient Approximation (GGA) and LDA by Kiejna et al..$^{36}$ yielded values of 0.61 and $0.66 \mathrm{eV}$, respectively, again in perfect agreement with our value of $0.69 \mathrm{eV}$. Previous studies reported $E_{\text {vac }}$ for Au to be in between 0.77 to $0.82 \mathrm{eV},{ }^{37}$ in accordance with $\mathrm{Li}$ et al., ${ }^{71}$ but slightly larger than our value of $0.58 \mathrm{eV}$. Other theoretical and experimental values for Au showed values between 0.82 and $0.93 \mathrm{eV}$, thus again slightly higher. ${ }^{38}$ We examined whether this discrepancy of $E_{\text {vac }}$ for a single atom was due to our $\mathrm{vdW}$ correction, yet the estimate without Grimme's correction yielded a similarly low value of $0.55 \mathrm{eV}$.

\section{$\mathrm{C}_{60}$ Adsorption}

In view of the high electron affinity of $\mathrm{C}_{60}$, a metal $\mathrm{C}_{60}$ charge transfer is expected, which leads to ionic binding in addition to possible covalent bonds. The abovementioned dissociation energy $D$ is defined as:

$$
D=T_{\text {сб }}+T(-v)-T\left(-v, C_{60}\right),
$$

where $T_{\text {сь }}$ is the total energy of $\mathrm{C}_{60}$ in vacuum (cell size dimensions of $20 \AA$ ) and $T\left(-v, C_{60}\right)$ the total energy of the surface with $v$ vacancies and $\mathrm{C}_{60}$ adsorbed upon. Results for $D$ are collected in in Table 3. For all considered materials and surface structures we obtain a high value of $D$ around or larger than $2 \mathrm{eV}$. The winner is gold with a particularly high adsorption energy whereas $\mathrm{Al}$ and Be share the second rank. In a previous study Tang et al. obtained, by DFT but without applying any dispersion correction, adsorption energies of $2.07,2.33$, and $2.56 \mathrm{eV}$ for single, triple, and septuple atom vacancies, respectively, as compared to a value of $1.2 \mathrm{eV}$ for a dense monolayer of $\mathrm{C}_{60}$ at clean $\mathrm{Au}(111) .^{\text {rd }}$ Note that in this work a denser fullerene monolayer was used, and, consequently, the reduced adsorption energies may well be the result of steric alexander 28/3/2014 8:28

Comentario [4]: AK: the bulk lattice constant is relaxed, but a bulk vacancy would lead also to relaxation, which is not included in the cohesive energy. The values are very similar however if this relaxation is also taken into account. I think it is too long and boring for the reader to write this here in more detail?

Fransis 12/4/2014 16:27

Comentario [5]: FV: Yes, maybe too long and boring. Let us drop it, and only put it in case a

referee asks for it. 
repulsions in between fullerenes. Other DFT works report adsorption strengths of the order of 1.9-2.2 eV for fullerene adsorbed on clean gold, ${ }^{\text {s. }}$ and classical force field models depicted binding strengths of 1.26 and $3.12 \mathrm{eV}$ for $\mathrm{C}_{60}$ on surface reconstructed grooves and dimples, respectively..$^{g_{8}}$

In any case vdW dispersion seems to play a major role in the attachment of fullerenes to metal surfaces, as had also been found for similar systems such as graphene. ${ }^{39}$ For example, $\mathrm{C}_{60}$ was found to adsorb on $\mathrm{Au}(111)$ with a hexagon parallel to the surface over an $h c p$ three-fold hollow site, ${ }^{,}$with a primarily covalent bond of $1.27 \mathrm{eV}$ strength, and a charge transfer of $0.2 \mathrm{e}$, according to DFT calculations without vdW description. A subsequent DFT study including $\mathrm{vdW}^{\mathrm{s}}$ showed that adsorption is dominated by dispersive forces, yet bonding remained covalent in nature. The optimized structure, close to the presently obtained one, implies a binding to the $f c c$ three-fold hollow site with binding strengths in the range of 1.71-2.97 eV depending on the method for dispersion correction.

As a general trend vacancies strongly enhance the adsorption energy. It first increases with the vacancy number $v$, but it may well happen that at some point the extension of the pit exceeds that of $\mathrm{C}_{60}$. Consequently, $D$ decreases again from $v=3$ to $v=7$ for $\mathrm{Al}$ and $\mathrm{Be}$. Note that the vdW interactions play a major role to enhance $D$; optimization without the Grimme correction resulted in rather small adsorption energies of $0.16 \mathrm{eV}$ for clean $\mathrm{Al}$ and $0.07 \mathrm{eV}$ for clean Be with $\mathrm{C}_{60}$ locating far away from the surface. A previous DFT study of $\mathrm{C}_{6} / \mathrm{Au}(111)$ without accounting for dispersion obtained a similarly low binding energy of $0.18 \mathrm{eV} .{ }^{94}$ However, the $\mathrm{C}_{60}$ desorption temperature of both $\mathrm{Au}(111)$ and $\mathrm{Al}(111)$ lies around $700 \mathrm{~K}$, suggesting a strong covalent bonding with the surface. ${ }^{\text {i.s }}$

Table 3: Dissociation energies $D$ (equivalent to the adsorption energy of $\mathrm{C}_{60}$ at the substrate), Bader charge transfer $\mathrm{q}_{\mathrm{c}}$, and number of covalent bonds $\mathrm{n}_{\mathrm{c} \text {. }}$.

\begin{tabular}{lcccccccccccc}
\hline & \multicolumn{4}{c}{$\mathbf{A l}(\mathbf{1 1 1})$} & \multicolumn{4}{c}{$\boldsymbol{A u}(\mathbf{1 1 1})$} & \multicolumn{5}{c}{$\operatorname{Be}(\mathbf{0 0 1})$} \\
\hline \hline $\boldsymbol{v}$ & $\mathbf{0}$ & $\mathbf{1}$ & $\mathbf{3}$ & $\mathbf{7}$ & $\mathbf{0}$ & $\mathbf{1}$ & $\mathbf{3}$ & $\mathbf{7}$ & $\mathbf{0}$ & $\mathbf{1}$ & $\mathbf{3}$ & $\mathbf{7}$ \\
$\boldsymbol{D} / \mathbf{e V}$ & 1.99 & 3.97 & 4.28 & 3.44 & 3.39 & 4.21 & 4.94 & 5.20 & 2.45 & 3.69 & 4.21 & 3.58 \\
$\mathbf{q}_{\mathrm{c}} / \mathbf{e}$ & -2.3 & -4.2 & -4.1 & -5.2 & -0.08 & -0.2 & -0.18 & -0.11 & -4.8 & -4.3 & -5.2 & -6.1 \\
$\mathbf{n}_{\text {св }}$ & 6 & 6 & 4 & 6 & 0 & 0 & 0 & 0 & 6 & 6 & 9 & 9 \\
\hline
\end{tabular}

A Bader electron density analysis reveals large charge transfer from $\mathrm{Al}$ and $\mathrm{Be}$ to $\mathrm{C}_{60}{ }^{40} \mathrm{It}$ increases dramatically with $v$ up to 6 electrons for $v=7$, that are transferred from $\mathrm{Be}$ to $\mathrm{C}_{6}$, 
although the electron affinity of isolated $\mathrm{C}_{60}$ is only positive up to $\mathrm{C}_{60}{ }^{2} \cdot{ }^{41}$ A completely different behavior is found for $\mathrm{Au}$ which retains the electrons, so the charge transfer is limited to 0.1-0.2 e. Previous ultraviolet photoelectron spectroscopy experiments estimated a charge transfer from gold to $\mathrm{C}_{\infty}$ of $1 \pm 0.2 \mathrm{e}$. This exceeds the value predicted by our as well as other DFT calculations by a large margin and remains unexplained so far. ${ }^{7 i_{0}}$

The true locations of charge accumulation and depletion are resolved in Charge Density Difference $(C D D)$ maps, see Figure 1. Again the bonding mechanism at the Au surface is completely different than the bonding at $\mathrm{Al}$ and $\mathrm{Be}$. For Au depletion regions that resemble welllocalized $p$-orbitals (blue lobes) are found, extending far inside the Au surface. The charge is transferred to the red-green clouds that might be interpreted as covalent bonds, as also found by Hamada et al. and Hinterstein et al. by DFT-based CDD plots showing the directional character of C-Au bonds. ${ }^{\text {ress }}$ Interestingly, the CDD plots do not show all these features without taking vdW into account. $\%$ We also looked at the Electron Localization Functions (ELF) and found no apparent covalent bonds for $\mathrm{C}_{60}$ on $\mathrm{Au}$ in contrast to $\mathrm{C}_{60}$ on either $\mathrm{Al}$ or $\mathrm{Be}$, where clear signatures of covalent bonding appear at the places of charge accumulation between $\mathrm{C}_{60}$ and the respective surface. In the case of Be, two $\mathrm{C}$ atoms are seen to share bonds to one surface atom. For Al, in contrast, every $\mathrm{C}$ atom is clearly bonded to one specific surface atom. This results in different optimized geometries because the bottom hexagon of $\mathrm{C}_{60}$ resides on top of an $\mathrm{Al}$ atom but in the center of three Be atoms. The number of covalent bonds $\mathrm{n}_{\text {св }}$ observed from ELF is given in Table 3 as well. The deeper the vacancies get, the deeper the fullerene dives into the surface, and different atoms become available for covalent bonding. The ELF covalent regions of the clean surface are much smaller than the ones in the presence of vacancies, mirroring the corresponding difference in $D$. 

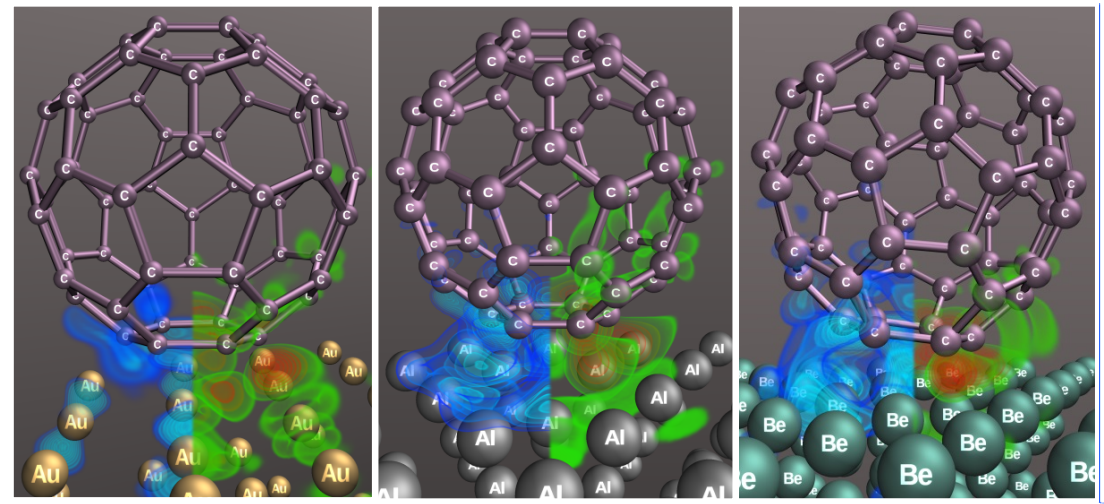

Figure 1: CDD volume rendering of regions with charge accumulation (green red = low high) and depletion (blue light blue = low high) due to adsorption of $\mathrm{C}_{60}$ for clean surfaces. For the sake of clarity very small charge differences are omitted.

The total Density Of States $(D O S)$ for the $\mathrm{C}_{60}$ adsorbed on $\mathrm{Al}$ system is projected on the $\mathrm{Al}$ atoms in Figure 2a and compared with the DOS of a pristine Al surface. Note that the amplitudes for $\mathrm{Al}+\mathrm{C}_{60}$ without and with the seven-atom vacancy were resized to meet the amplitudes of the pristine Al DOS at low energy. Small changes appear both below and above the Fermi level $\left(\mathrm{E}_{\mathrm{F}}\right)$ upon absorption of $\mathrm{C}_{60}$, where the discrete states of $\mathrm{C}_{60}$ are superposed with the quasi-continuum of the surface. The DOS is smeared out due to the seven-atom vacancy and further by the presence of $\mathrm{C}_{60}$. The same comparison for Au yields a similar picture with a much lower degree of smearing, while for Be the DOS is relatively unaffected by adsorption of $\mathrm{C}_{60}$ and vacancy formation. These observations are in accordance with the fact that $\mathrm{Al}$ mostly deforms in response to vacancy formation and $\mathrm{C}_{60}$ adsorption.
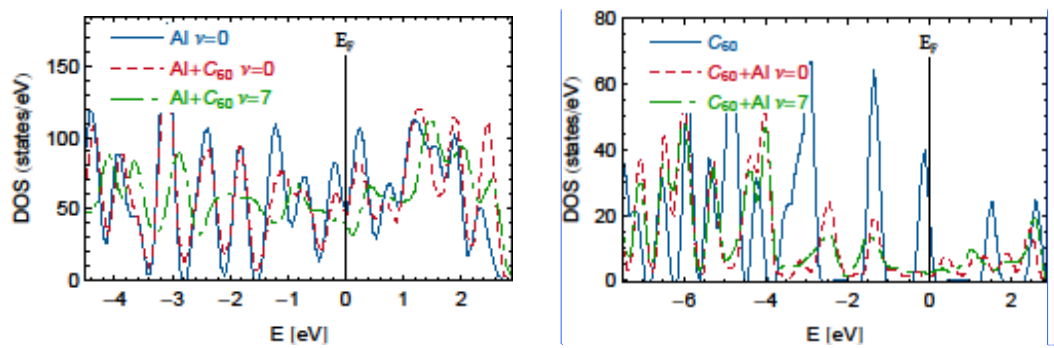

\section{alexander 12/4/2014 16:31}

Comentario [7]: FV OK, but when making pdf out of it let us make sure that they are perfectly
visible.

Figure 2: DOS of Aluminum in $\mathrm{C}_{60}+\mathrm{Al}(v)$ compared to the clean $\mathrm{Al}$ surface (a) and DOS of carbon in $\mathrm{C}_{60}+\mathrm{Al}(v)$ compared to $\mathrm{C}_{60}$ in vacuum $(b)$. 
The scaled DOS projected on carbon atoms is shown in Figure $2 \mathrm{~b}$; compared to $\mathrm{C}_{60}$ in vacuum the $\mathrm{C}_{60}$ states when adsorbed on $\mathrm{Al}$ are shifted to lower energies by $\sim 1 \mathrm{eV}$. Furthermore, in the vicinity of $E_{\mathrm{F}}$ the sharp peaks of the molecule are tremendously smeared out, yielding a metallic behavior, and, even so, a small part of the LUMO is shifted below $E_{F}$, in accordance with previous DFT calculations. ${ }^{90}$ This effect is stronger when $\mathrm{C}_{80}$ is adsorbed to a vacancy than on the clean surface. Largely similar observations are made for the cases of $\mathrm{Au}$ and Be. The findings are consistent with previous Scanning Tunneling Spectroscopy (STS) experiments on $\mathrm{Au}(110)$, which revealed that a significant fraction of $\mathrm{C}_{60}$ LUMO broadens and shifts downwards towards the Fermi level. . $^{\text {sa }}$

\section{Discussion}

We first discuss fullerene adsorption induced vacancy formation. Upon adsorption, $D$ is released, and this can give rise to vacancy formation, since $D(v)>E_{v a}(v)$ for all structures considered here except for septuple-atom vacancies for $\mathrm{Al}$ and Be. However, other adsorption energy dissipation channels exist. A better suited criterion to determine whether a vacancy formation is thermodynamically allowed is that the $D$ energy on a vacancy must surpass that on the clean surface plus the vacancy-adatom formation energy: $D(v)>D(0)+E_{v a}(v)$. This ensures the adsorption-on-a-vacancy state to be the ground state, being of higher stability than the adsorption on the pristine surface. The latter more strict criterion is only fulfilled for $\mathrm{Al}(111)$ single-atom vacancies, where a total energy gain of $0.38 \mathrm{eV}$ is recorded. However, the missing energy for the other structures is not very high $(0.29 \mathrm{eV}$ for $\mathrm{Au}$ and $0.85 \mathrm{eV}$ for Be in the case of the single-atom vacancy) and therefore could easily be supplied, for instance, by addition of the adatom nearby $\mathrm{C}_{60}$, or by forming surface dimers, or even larger clusters. In addition, adsorbate location at step edges could gain extra energy ${ }^{7,3,33}$ given the enhanced adsorption-ability of surface under-coordinated metal atoms located at edges between terraces..$^{42}$ Besides, the small energy cost could be thermally supplied. Overall, adsorption of $\mathrm{C}_{60}$ reduces the cost of vacancy formation compared to pristine surfaces. This clearly points for the formation of surface metal vacancies that follow the pattern of $\mathrm{C}_{60}$ adsorbates. The concept is backed up by STM experiments which showed bright and $\operatorname{dim} \mathrm{C}_{60}$ on $\mathrm{Au}(111)$, where dim molecules were identified to reside on top of multiatom vacancies. ${ }^{7 c}$ This is an example of how the adsorption of a $\mathrm{C}_{60}$ monolayer yielded a periodic ordering of adsorption-induced vacancies on the surface.

The conditions for $\mathrm{C}_{60} \mathrm{CSA}$ are more difficult to assess. One can propose a Gedankenexperiment involving a metal surface with already well-separated vacancies and a temperature large enough to allow for $\mathrm{C}_{60}$ diffusion over vacancy-free surface areas. Thus, the first deposited $\mathrm{C}_{60}$ can move freely on the clean surface until it finds a vacancy. A recent study 
estimated an activation energy of $13 \mathrm{meV}$ for single fullerene diffusion on $\mathrm{Au}(111)$; $^{\text {pa }^{a}}$ such a small activation energy would allow $\mathrm{C}_{60}$ to easily find vacancies, supporting the CSA feasibility. Since the binding energy on the vacancy is by $1-2 \mathrm{eV}$ larger than on the clean surface, the $\mathrm{C}_{60}$ will remain anchored there unless the temperature is too high. A second $\mathrm{C}_{60}$ has two possible fates. Either it moves nearby to the first $\mathrm{C}_{60}$ and initializes cluster nucleation, releasing a few hundreds of $\mathrm{meV}_{, 33}$ or it finds an unoccupied vacancy, releasing a much higher margin of energy. From an energetic point of view there would be a temperature window, where nucleation is suppressed because the binding to other $\mathrm{C}_{60}$ is not strong enough, while simultaneously $\mathrm{C}_{60}$ forms stable bonds to vacancies. The above-introduced competing process of induced vacancy formation could, however, complicate this picture. We conclude that with accurate temperature control, the selfassembly on pre-structured template surfaces - containing surface vacancies - could be controlled, leading to user-defined patterns of adsorbate molecules, given that adsorptioninduced vacancy formation is energetically unfavorable, such as on $\mathrm{Au}(111)$ and $\mathrm{Be}(001)$.

Previous STM experiments of $\mathrm{C}_{6} / \mathrm{Au}(111)$ at RT assisted by DFT calculations also revealed vacancies as preferred bonding sites..$^{74}$ At $46 \mathrm{~K} \mathrm{C}_{60}$ molecules seem to attach to the elbow sites of the $\mathrm{Au}(111)$ herringbone reconstruction. When annealing to RT these sites are vacated, and nucleation at step edges occurs without vacancy formation. When starting directly at RT, Tang et al. found individual molecules at elbow sites and further cluster nucleation in between them upon further deposition, whereas at low temperature clusters only nucleated around single molecules adsorbed at elbow sites. This controversy led the authors to argue that vacancies form below $\mathrm{C}_{60}$ to stabilize its adsorption at RT. The hypothesis was supported by a $\mathrm{C}_{60}$ height of $6.2 \AA$ at $46 \mathrm{~K}$ while at RT $\mathrm{C}_{60}$ lies $2.2 \AA$ deeper. This 'sinking' is in agreement with the adsorption over seven-atom pits, and demonstrates that $\mathrm{C}_{60}$ can be safely stored over vacancies at $\mathrm{RT}$, supporting the idea of the CSA process that will be necessary for envisaged nanodevices. XRD studies also showed surface reconstruction with pit formation to accommodate $\mathrm{C}_{\infty}$ on $\mathrm{Au}(110)$, , $^{\text {and }}$ an herringbone $\mathrm{Au}(111) .^{\text {ㅎ }}$ The latter displayed $\mathrm{C}$ - $\mathrm{Au}$ distances of $2.49 \AA$ with a $\mathrm{C}_{60}$ hexagon facing the single-atom vacancy, close to our value of $2.33 \AA$, indicating a mixed covalent/ionic bonding.

As far as aluminum is concerned, the $\mathrm{Al}(111)$ surface also reconstructs under a compressed $\mathrm{C}_{60}$ overlayer. ${ }^{7 \mathrm{~g}^{\mathrm{j}}}$ Stengel et al. proposed that two Al adatoms from single-atom vacancies under two out of three adsorbed $\mathrm{C}_{60}$ form a dimer that stays in the interstitial region between $\mathrm{C}_{60}$ molecules, further stabilizing the system..$^{78}$ The structure naturally explains why one out of three $\mathrm{C}_{60}$ protrudes from the surface. The authors gave adsorption energies ranging 0.98$1.38 \mathrm{eV}$, yet not accounting for $\mathrm{vdW}$ forces, and estimated a total energy gain due to the reconstruction of $0.8 / 3=0.27 \mathrm{eV}$ per $\mathrm{C}_{\infty}$ molecule. In another related study ${ }^{n}$ LDA DFT calculations yielded adsorption energies on pristine surfaces of 1.37, 1.25, and $1.52 \mathrm{eV}$ for $\mathrm{Al}$, 
$\mathrm{Au}$, and $\mathrm{Ag}$, respectively. On vacancies the adsorption energies increased to 2.34, 2.69, and 2.61

$\mathrm{eV}$, respectively. The authors also contemplated structures where vacancy atoms occupy the free

space in between $\mathrm{C}_{60}$. Using the unreconstructed surface as energy reference, the adsorption energies are $1.40,1.35$, and $1.59 \mathrm{eV}$. Here the energy needed to create a vacancy is accounted for, and still these adsorption energies are by $0.10,0.03$, and $0.07 \mathrm{eV}$ larger than the adsorption energies found on clean surfaces. This study suggests $\mathrm{C}_{60}$-induced vacancy formation on all considered surfaces, and is fully compatible with our results for aluminum.

No literature values were found for beryllium which turned out to be most resistant with respect to vacancy formation among the three metals investigated in this work. The stability of Be surface could be useful for the CSA process.

\section{Conclusion}

We studied the energetic criteria for vacancy formation and fullerene adsorption on gold, aluminum and beryllium. To this end we employed density functional theory calculations taking van der Waals interactions into account. We found that the cost for vacancy formation increases in the order $\mathrm{Au}<\mathrm{Al}<\mathrm{Be}$. A strong adsorption of $\mathrm{C}_{60}$ is found for all metals, surpassing dissociation energies of $2 \mathrm{eV}$. Adsorption on surface vacancies is enhanced compared to pristine surfaces, so that $\mathrm{C}_{\infty}$ molecules might occupy user-defined patterns of vacancies (vacancy patterning). On the other hand, the adsorption energy on an $\mathrm{Al}$ vacancy is higher than for adsorption on the pristine surface plus the vacancy costs;, only a slight endothermicity is found for $\mathrm{Au}$ and $\mathrm{Be}$. This suggests the possibility of patterning vacancies when adsorbing $\mathrm{C}_{60}$ on these surfaces. The computational results are backed up with available experimental evidence, and future applications are envisaged for such a patterning in nanodevices. The fact that Be is more resilient to surface vacancy formations than Al should be kept in mind when comparing the sputtering properties of these metals.

\section{Acknowledgements}

Part of this work was supported by the Austrian Ministry of Science BMWF as part of the UniInfrastrukturprogramm of the Research Platform Scientific Computing at the University of Innsbruck and funded by the Austrian Science Fund (FWF) DK+ project Computational Interdisciplinary Modeling, W1227-N16. This work was also supported by the Spanish MICINN grant CTQ2012-30751, and, in part, by the Generalitat de Catalunya (grants 2009SGR1041 and XRQTC). F.V. thanks the MINECO for the postdoctoral Juan de la Cierva and Ramón y Cajal 
grants (JCI-2010-06372, RYC-2012-10129), F.I. acknowledges additional funding through the 2009 ICREA Academia award for excellence in university research. F.H. acknowledges support from the Tennessee NSF-EPSCoR Grant TN-SCORE (NSF EPS 1004083). Support by the European Commission under the Contract of Association between EURATOM and the Austrian Academy of Sciences within the framework of the European Fusion Development Agreement is also acknowledged.

\section{References}

1. Schierbaum, K. D.; Weiss, T.; van Veizen, E. U. T.; Engbersen, J. F. J.; Reinhoudt, D. N.; Göpel, W., Molecular Recognition by Self-Assembled Monolayers of Cavitand Receptors. Science 1994, 265 (5177), 1413-1415.

2. Menard, E.; Meitl, M. A.; Sun, Y.; Park, J.-U.; Shir, D. J.-L.; Nam, Y.-S.; Jeon, S.; Rogers, J. A., Micro- and Nanopatterning Techniques for Organic Electronic and Optoelectronic Systems. Chem. Rev. 2007, 107 (4), 1117-1160.

3. Díez-Pérez, I.; Luna, M.; Teherán, F.; Ogletree, D. F.; Sanz, F.; Salmeron, M., Interaction of Water with Self-Assembled Monolayers of Alkylsilanes on Mica. Langmuir 2004, 20 (4), 1284-1290.

4. Kozlov, S. M.; Viñes, F.; Görling, A., Bandgap Engineering of Graphene by Physisorbed Adsorbates. Advanced Materials 2011, 23 (22-23), 2638-2643.

5. Vettiger, P.; Cross, G.; Despont, M.; Drechsler, U.; Durig, U.; Gotsmann, B.; Haberle, W.; Lantz, M. A.; Rothuizen, H. E.; Stutz, R.; Binnig, G. K., The "millipede" nanotechnology entering data storage. IEEE Trans. Nanotechnol. 2002, 1 (1), 39-55.

6. Vayssilov, G. N.; Lykhach, Y.; Migani, A.; Staudt, T.; Petrova, G. P.; Tsud, N.; Skála, T.; Bruix, A.; Illas, F.; Prince, K. C.; Matolı́n, V.r.r.; Neyman, K. M.; Libuda, J., Support nanostructure boosts oxygen transfer to catalytically active platinum nanoparticles. Nat Mater 2011, 10 (4), 310-315.

7. (a) Gimzewski, J. K.; Modesti, S.; Schlittler, R. R., Cooperative self-assembly of Au atoms and $\mathrm{C}_{60}$ on $\mathrm{Au}(110)$ surfaces. Phys. Rev. Lett. 1994, 72 (7), 1036-1039; (b) Torrelles, X.; Pedio, M.; Cepek, C.; Felici, R., $(2 \sqrt{3} \times 2 \sqrt{3}) R 30^{\circ}$ induced self-assembly ordering by $\mathrm{C}_{60}$ on a $\mathrm{Au}(111)$ surface: X-ray diffraction structure analysis. Phys. Rev. B 2012, 86 (7), 075461; (c) Tang, L.; Xie, Y.; Guo, Q., Complex orientational ordering of $\mathrm{C}_{\infty}$ molecules on Au(111). J. Chem. Phys. 2011, 135 (11), 114702; (d) Tang, L.; Zhang, X.; Guo, Q.; Wu, Y.-N.; Wang, L.-L.; Cheng, H.-P., Two bonding configurations for individually adsorbed $\mathrm{C}_{60}$ molecules on $\mathrm{Au}(111)$. Phys. Rev. B 2010, 82 (12), 125414; (e) Hinterstein, M.; Torrelles, X.; Felici, R.; Rius, J.; Huang, M.; Fabris, S.; Fuess, H.; Pedio, M., Looking underneath fullerenes on $\mathrm{Au}(110)$ : Formation of dimples in the substrate. Phys. Rev. B 2008, 77 (15), 153412; (f) Hoogenboom, B. W.; Hesper, R.; Tjeng, L. H.; Sawatzky, G. A., Charge transfer and doping-dependent hybridization of $\mathrm{C}_{60}$ on noble metals. Phys. Rev. B 1998, 57 (19), 11939-11942; (g) Stengel, M.; Vita, A. D.; Baldereschi, A., Adatom-Vacancy Mechanisms for the $\mathrm{C}_{\mathrm{co}} / \mathrm{Al}(111)-(6 \times 6)$ Reconstruction. Phys. Rev. Lett. 2003, 91 (16), 166101; (h) Johansson, M. K. J.; Maxwell, A. J.; Gray, S. M.; Brühwiler, P. A.; Mancini, D. C.; Johansson, L. S. O.; Mårtensson, N., Scanning tunneling microscopy of $\mathrm{C}_{60} / \mathrm{Al}(111)-6 \times 6$ : Inequivalent molecular sites and electronic structures. Phys. Rev. B 1996, 54 (19), 13472-13475; (i) Maxwell, A. J.; Brühwiler, P. A.; Arvanitis, D.; Hasselström, J.; Johansson, M. K. J.; Mårtensson, N., Electronic and geometric structure of $\mathrm{C}_{60}$ on $\mathrm{Al}(111)$ and $\mathrm{Al}(110)$. Phys. Rev. B 1998, 57 (12), 73127326; (j) Maxwell, A. J.; Brühwiler, P. A.; Andersson, S.; Arvanitis, D.; Hernnäs, B.; Karis, O.; Mancini, D. C.; Mårtensson, N.; Gray, S. M.; Johansson, M. K. J.; Johansson, L. S. O., $\mathrm{C}_{60}$ on $\mathrm{Al}(111)$ : Covalent bonding and surface reconstruction. Phys. Rev. B 1995, 
52 (8), R5546-R5549; (k) Felici, R.; Pedio, M.; Borgatti, F.; Iannotta, S.; Capozi, M.; Ciullo, G.; Stierle, A., X-ray-diffraction characterization of $\operatorname{Pt}(111)$ surface nanopatterning induced by $\mathrm{C}_{60}$ adsorption. Nat. Mater. 2005, 4 (9), 688-692; (1) Li, H. I.; Pussi, K.; Hanna, K. J.; Wang, L. L.; Johnson, D. D.; Cheng, H. P.; Shin, H.; Curtarolo, S.; Moritz, W.; Smerdon, J. A.; McGrath, R.; Diehl, R. D., Surface Geometry of $\mathrm{C}_{60}$ on Ag(111). Phys. Rev. Lett. 2009, 103 (5), 056101.

8. Tzeng, C. T.; Lo, W. S.; Yuh, J. Y.; Chu, R. Y.; Tsuei, K. D., Photoemission, near-edge $\mathrm{X}$-ray-absorption spectroscopy, and low-energy electron-diffraction study of $\mathrm{C}_{60}$ on Au(111) surfaces. Phys. Rev. B 2000, 61 (3), 2263-2272.

9. (a) Akimov, A. V.; Williams, C.; Kolomeisky, A. B., Charge Transfer and Chemisorption of Fullerene Molecules on Metal Surfaces: Application to Dynamics of Nanocars. $J$. Phys. Chem. C 2012, 116 (25), 13816-13826; (b) Hamada, I.; Tsukada, M., Adsorption of $\mathrm{C}_{\infty}$ on $\mathrm{Au}(111)$ revisited: A van der Waals density functional study. Phys. Rev. B 2011, 83 (24), 245437; (c) Gardener, J. A.; Briggs, G. A. D.; Castell, M. R., Scanning tunneling microscopy studies of $\mathrm{C}_{60}$ monolayers on $\mathrm{Au}(111)$. Phys. Rev. B 2009, 80 (23), 235434; (d) Abad, E.; Ortega, J.; Dappe, Y. J.; Flores, F., Dipoles and band alignment for benzene/Au(111) and $\mathrm{C}_{6} / \mathrm{Au}(111)$ interfaces. Appl. Phys. A 2009, 95 (1), 119-124; (e) Wang, L.-L.; Cheng, H.-P., Density functional study of the adsorption of a $\mathrm{C}_{\infty}$ monolayer on $\mathrm{Ag}(111)$ and $\mathrm{Au}(111)$ surfaces. Phys. Rev. B 2004, 69 (16), 165417; (f) Lu, X.;

Grobis, M.; Khoo, K. H.; Louie, S. G.; Crommie, M. F., Charge transfer and screening in individual $\mathrm{C}_{60}$ molecules on metal substrates:A scanning tunneling spectroscopy and theoretical study. Phys. Rev. B 2004, 70 (11), 115418; (g) Baxter, R. J.; Rudolf, P.; Teobaldi, G.; Zerbetto, F., Modelling of the Adsorption of $C_{60}$ on the Au(110) Surface. ChemPhysChem 2004, 5 (2), 245-248; (h) Nouchi, R.; Kanno, I., Resistance measurements of conducting $\mathrm{C}_{60}$ monolayers formed on $\mathrm{Au}$ and $\mathrm{Cu}$ films. J. Appl. Phys. 2003, 94 (5), 3212-3215; (i) Rogero, C.; Pascual, J. I.; Gomez-Herrero, J.; Baro, A. M., Resolution of site-specific bonding properties of $\mathrm{C}_{60}$ adsorbed on $\mathrm{Au}(111)$. J. Chem. Phys. 2002, 116 (2), 832-836; (j) Pedio, M.; Felici, R.; Torrelles, X.; Rudolf, P.; Capozi, M.; Rius, J.; Ferrer, S., Study of $\mathrm{C}_{6} / \mathrm{Au}(110)-\mathrm{p}(6 \times 5)$ Reconstruction from In-Plane X-Ray Diffraction Data. Phys. Rev. Lett. 2000, 85 (5), 1040-1043; (k) Modesti, S.; Cerasari, S.; Rudolf, P., Determination of charge states of $\mathrm{C}_{60}$ adsorbed on metal surfaces. Phys. Rev. Lett. 1993, 71 (15), 2469-2472; (1) Kuk, Y.; Kim, D. K.; Suh, Y. D.; Park, K. H.; Noh, H. P.; Oh, S. J.; Kim, S. K., Stressed $\mathrm{C}_{6}$ layers on $\mathrm{Au}(001)$. Phys. Rev. Lett. 1993, 70 (13), 1948-1951; (m) Weaver, M. J.; Gao, X.; Zhang, Y., Scanning tunneling microscopy of carbon molecule $\left(\mathrm{C}_{60}\right.$ and $\left.\mathrm{C}_{50}\right)$ on ordered gold (111) and gold (110): molecular structure and electron transmission. J. Phys. Chem. 1992, 96 (2), 510-513; (n) Altman, E. I.; Colton, R. J., Nucleation, growth, and structure of fullerene films on Au(111). Surf. Sci. 1992, 279 (1-2), 49-67; (o) Wilson, R. J.; Meijer, G.; Bethune, D. S.; Johnson, R. D.; Chambliss, D. D.; de Vries, M. S.; Hunziker, H. E.; Wendt, H. R., Imaging $\mathrm{C}_{60}$ clusters on a surface using a scanning tunnelling microscope. Nature 1990, 348 (6302), 621-622.

10. (a) Zhang, X.; He, W.; Zhao, A.; Li, H.; Chen, L.; Pai, W. W.; Hou, J.; Loy, M. M. T.; Yang, J.; Xiao, X., Geometric and electronic structure of a $\mathrm{C}_{60}$ monolayer on $\mathrm{Ag}(100)$. Phys. Rev. B 2007, 75 (23), 235444; (b) Pedio, M.; Hevesi, K.; Zema, N.; Capozi, M.; Perfetti, P.; Gouttebaron, R.; Pireaux, J. J.; Caudano, R.; Rudolf, P., Co/metal surfaces: adsorption and decomposition. Surf. Sci. 1999, 437 (1-2), 249-260; (c) David, T.; Gimzewski, J. K.; Purdie, D.; Reihl, B.; Schlittler, R. R., Epitaxial growth of $C_{6}$ on $\operatorname{Ag}(110)$ studied by scanning tunneling microscopy and tunneling spectroscopy. Phys. Rev. B 1994, 50 (8), 5810-5813.

11. Kiguchi, M.; Iizumi, K.-i.; Saiki, K.; Koma, A., Atomic and electronic structures of heteroepitaxial $\mathrm{C}_{60}$ film grown on $\mathrm{Ni}\left(\begin{array}{lll}1 & 1 & 1\end{array}\right), \mathrm{Cu}\left(\begin{array}{lll}1 & 1 & 1\end{array}\right)$. Appl. Surf. Sci. 2003, 212-213 (0), 101-104.

12. Weckesser, J.; Barth, J. V.; Kern, K., Mobility and bonding transition of $\mathrm{C}_{60}$ on $\operatorname{Pd}(110)$. Phys. Rev. B 2001, 64 (16), 161403. 
13. (a) Liu, C.; Qin, Z.; Chen, J.; Guo, Q.; Yu, Y.; Cao, G., Molecular orientations and interfacial structure of $\mathrm{C}_{60}$ on $\mathrm{Pt}(111)$. J. Chem. Phys. 2011, 134 (4), 044707-6; (b)

Torrelles, X.; Langlais, V.; De Santis, M.; Tolentino, H. C. N.; Gauthier, Y., Nanoscale

Patterning by $\mathrm{C}_{60}$ Ordering on Pt(110). J. Phys. Chem. C 2010, 114 (37), 15645-15652; (c)

Sogo, M.; Sakamoto, Y.; Aoki, M.; Masuda, S.; Yanagisawa, S.; Morikawa, Y., C

Adsorbed on Platinum Surface: A Good Mediator of Metal Wave Function. J. Phys.

Chem. C 2010, 114 (8), 3504-3506; (d) Orzali, T.; Forrer, D.; Sambi, M.; Vittadini, A.;

Casarin, M.; Tondello, E., Temperature-Dependent Self-Assemblies of $\mathrm{C}_{\text {ь }}$ on $(1 \times 2)$ -

Pt(110): A STM/DFT Investigation. J. Phys. Chem. C 2007, 112 (2), 378-390; (e)

Casarin, M.; Forrer, D.; Orzali, T.; Petukhov, M.; Sambi, M.; Tondello, E.; Vittadini, A., Strong Bonding of Single $\mathrm{C}_{60}$ Molecules to $(1 \times 2)-\mathrm{Pt}(110)$ : an STM/DFT Investigation. J. Phys. Chem.C 2007, 111 (26), 9365-9373; (f) Swami, N.; He, H.; Koel, B. E., Polymerization and decomposition of $\mathrm{C}_{60}$ on Pt(111) surfaces. Phys. Rev. B 1999, 59 (12), 8283-8291; (g) He, H.; Swami, N.; Koel, B. E., Control of the growth of ordered C ${ }_{60}$ films by chemical modification of Pt(111) surfaces. Thin Solid Films 1999, 348 (1-2), 30-37.

14. (a) Pai, W. W.; Jeng, H. T.; Cheng, C. M.; Lin, C. H.; Xiao, X.; Zhao, A.; Zhang, X.; Xu, G.; Shi, X. Q.; Van Hove, M. A.; Hsue, C. S.; Tsuei, K. D., Optimal Electron Doping of a $\mathrm{C}_{6}$ Monolayer on $\mathrm{Cu}(111)$ via Interface Reconstruction. Phys. Rev. Lett. 2010, 104 (3), 036103; (b) Tamai, A.; Seitsonen, A. P.; Baumberger, F.; Hengsberger, M.; Shen, Z. X.; Greber, T.; Osterwalder, J., Electronic structure at the $\mathrm{C}_{60} /$ metal interface: An angleresolved photoemission and first-principles study. Phys. Rev. B 2008, 77 (7), 075134; (c) Wang, L.-L.; Cheng, H.-P., Rotation, translation, charge transfer, and electronic structure of $\mathrm{C}_{60}$ on $\mathrm{Cu}(111)$ surface. Phys. Rev. B 2004, 69 (4), 045404; (d) Silien, C.; Pradhan, N. A.; Ho, W.; Thiry, P. A., Influence of adsorbate-substrate interaction on the local electronic structure of $\mathrm{C}_{60}$ studied by low-temperature STM. Phys. Rev. B 2004, 69 (11), 115434; (e) Ogawa, A.; Tachibana, M.; Kondo, M.; Yoshizawa, K.; Fujimoto, H.; Hoffmann, R., Orbital Interactions between a $\mathrm{C}_{60}$ Molecule and $\mathrm{Cu}(111)$ Surface. J. Phys . Chem. B 2003, 107 (46), 12672-12679; (f) Fasel, R.; Aebi, P.; Agostino, R. G.; Naumović, D.; Osterwalder, J.; Santaniello, A.; Schlapbach, L., Orientation of Adsorbed $\mathrm{C}_{60}$ Molecules Determined via X-Ray Photoelectron Diffraction. Phys. Rev. Lett. 1996, 76 (25), 4733-4736; (g) Maruyama, Y.; Ohno, K.; Kawazoe, Y., Electronic structures of $C_{\infty}$ and $\mathrm{C}_{70}$ adsorbed on the $\mathrm{Cu}(111)$ surface and intramolecular STM images. Phys. Rev. B 1995, 52 (3), 2070-2075; (h) Hashizume, T.; Motai, K.; Wang, X. D.; Shinohara, H.; Saito, Y.; Maruyama, Y.; Ohno, K.; Kawazoe, Y.; Nishina, Y.; Pickering, H. W.; Kuk, Y.; Sakurai, T., Intramolecular structures of $\mathrm{C}_{60}$ molecules adsorbed on the $\mathrm{Cu}(111)-(1 \times 1)$ surface. Phys. Rev. Lett. 1993, 71 (18), 2959-2962.

15. (a) Hou, J. G.; Yang, J.; Wang, H.; Li, Q.; Zeng, C.; Lin, H.; Bing, W.; Chen, D. M.; Zhu, Q., Identifying Molecular Orientation of Individual $C_{60}$ on a Si(111)-(7×7) Surface. Phys. Rev. Lett. 1999, 83 (15), 3001-3004; (b) Li, Y. Z.; Chander, M.; Patrin, J. C.; Weaver, J. H.; Chibante, L. P. F.; Smalley, R. E., Adsorption of individual $\mathrm{C}_{60}$ molecules on Si(111). Phys. Rev. B 1992, 45 (23), 13837-13840.

16. (a) Barth, J. V.; Brune, H.; Ertl, G.; Behm, R. J., Scanning tunneling microscopy observations on the reconstructed $\mathrm{Au}(111)$ surface: Atomic structure, long-range superstructure, rotational domains, and surface defects. Phys. Rev. B 1990, 42 (15), $9307-$ 9318; (b) Allmers, T.; Donath, M., Growth and morphology of thin Fe films on flat and vicinal Au(111): a comparative study. New J. Phys. 2009, 11 (10), 103049.

17. (a) Li, G.; Shrotriya, V.; Huang, J.; Yao, Y.; Moriarty, T.; Emery, K.; Yang, Y., Highefficiency solution processable polymer photovoltaic cells by self-organization of polymer blends. Nat. Mater. 2005, 4 (11), 864-868; (b) Clarke, T. M.; Durrant, J. R., Charge Photogeneration in Organic Solar Cells. Chem. Rev. 2010, 110 (11), 6736-6767.

18. (a) Federici, G., Plasma wall interactions in ITER. Physica Scripta 2006, 2006 (T124), 1; (b) Maihom, T.; Sukuba, I.; Janev, R.; Becker, K.; Märk, T.; Kaiser, A.; Limtrakul, J.; Urban, J.; Mach, P.; Probst, M., Electron impact ionization cross sections of beryllium and beryllium hydrides. Eur. Phys. J. D 2013, 67 (1), 1-5. 
19. (a) Kresse, G.; Furthmüller, J., Efficient iterative schemes for ab initio total-energy calculations using a plane-wave basis set. Phys. Rev. B 1996, 54 (16), 11169-11186; (b) Kresse, G.; Furthmüller, J., Efficiency of ab-initio total energy calculations for metals and semiconductors using a plane-wave basis set. Computational Materials Science 1996, 6 (1), 15-50; (c) Kresse, G.; Hafner, J., Ab initio molecular dynamics for liquid metals. Phys. Rev. B 1993, 47 (1), 558-561; (d) Kresse, G.; Hafner, J., Ab initio moleculardynamics simulation of the liquid-metal-amorphous-semiconductor transition in germanium. Phys. Rev. B 1994, 49 (20), 14251-14269.

20. (a) Perdew, J. P.; Burke, K.; Ernzerhof, M., Generalized Gradient Approximation Made Simple. Phys. Rev. Lett. 1996, 77 (18), 3865-3868; (b) Blöchl, P. E., Projector augmented-wave method. Phys. Rev. B 1994, 50 (24), 17953-17979; (c) Grimme, S., Semiempirical GGA-type density functional constructed with a long-range dispersion correction. J. Comp. Chem. 2006, 27 (15), 1787-1799.

21. Amft, M.; Sébastien, L.; Eriksson, O.; Skorodumova, N. V., Adsorption of Cu, Ag, and $\mathrm{Au}$ atoms on graphene including van der Waals interactions. J. Phys.: Condens. Matter 2011, 23 (39), 395001.

22. Mineralogy Database. http://www.webmineral.com/ (accessed 10/8).

23. Gaudoin, R.; Foulkes, W. M. C.; Rajagopal, G., Ab initio calculations of the cohesive energy and the bulk modulus of aluminium. J. Phys.: Condens. Matter 2002, 14 (38), 8787 .

24. (a) Fall, C. J.; Binggeli, N.; Baldereschi, A., Anomaly in the anisotropy of the aluminum work function. Phys. Rev.B 1998, 58 (12), R7544-R7547; (b) Skriver, H. L.; Rosengaard, N. M., Surface energy and work function of elemental metals. Phys. Rev. B 1992, 46 (11), 7157-7168.

25. Schöchlin, J.; Bohnen, K. P.; Ho, K. M., Structure and dynamics at the Al(111)-surface. Surf. Sci. 1995, 324 (2-3), 113-121.

26. Chan, B.; Yim, W.-L., Accurate Computation of Cohesive Energies for Small to Medium-Sized Gold Clusters. J. Chem. Theory Comput. 2013, 9 (4), 1964-1970.

27. (a) Anderson, P. A., Work Function of Gold. Physical Review 1959, 115 (3), 553-554; (b) Fall, C. J.; Binggeli, N.; Baldereschi, A., Work-function anisotropy in noble metals: Contributions from d states and effects of the surface atomic structure. Phys. Rev. B 2000, $61(12), 8489-8495$.

28. Needs, R. J.; Mansfield, M., Calculations of the surface stress tensor and surface energy of the (111) surfaces of iridium, platinum and gold. J. Phys.: Condens. Matter 1989, I (41), 7555 .

29. Hao, A.; Zhu, Y., First-principle investigations of structural stability of beryllium under high pressure. J. Appl. Phys. 2012, 112 (2), 023519-4.

30. Chou, M. Y.; Lam, P. K.; Cohen, M. L., Ab initio study of structural and electronic properties of beryllium. Phys. Rev. B 1983, 28 (8), 4179-4185.

31. (a) Benger, W.; Ritter, G.; Heinzl, R., The Concepts of VISH. In 4th High-End Visualization Workshop, Obergurgl, Tyrol, Austria, June 18-21, 2007, Berlin, Lehmanns Media-LOB.de: 2007; (b) Kaiser, A.; Ismailova, O.; Koskela, A.; Huber, S. E.; Ritter, M.; Cosenza, B.; Benger, W.; Nazmutdinov, R.; Probst, M., Ethylene glycol revisited: Molecular dynamics simulations and visualization of the liquid and its hydrogen-bond network. J. Mol. Liqu. 2014, 189 (0), 20-29.

32. Stoltze, P., Simulation of surface defects. J. Phys.: Condens. Matter 1994, 6 (45), 9495.

33. Stumpf, R.; Scheffler, M., Ab initio calculations of energies and self-diffusion on flat and stepped surfaces of $\mathrm{Al}$ and their implications on crystal growth. Phys. Rev. B 1996, 53 (8), 4958-4973.

34. Barbalace, K. Periodic Table of Elements. EnvironmentalChemistry.com. 1995 - 2014. http://environmentalchemistry.com/yogi/periodic/ (accessed 03/36).

35. Polatoglou, H. M.; Methfessel, M.; Scheffler, M., Vacancy-formation energies at the (111) surface and in bulk Al, Cu, Ag, and Rh. Phys. Rev. B 1993, 48 (3), 1877-1883. 
36. Kiejna, A., Vacancy formation and $\mathrm{O}$ adsorption at the $\mathrm{Al}(111)$ surface. Phys. Rev. B 2003, 68 (23), 235405.

37. Korzhavyi, P. A.; Abrikosov, I. A.; Johansson, B.; Ruban, A. V.; Skriver, H. L., Firstprinciples calculations of the vacancy formation energy in transition and noble metals. Phys.Rev.B 1999, 59 (18), 11693-11703.

38. Korhonen, T.; Puska, M. J.; Nieminen, R. M., Vacancy-formation energies for fcc and bcc transition metals. Phys. Rev. B 1995, 51 (15), 9526-9532.

39. Kozlov, S. M.; Viñes, F.; Görling, A., Bonding Mechanisms of Graphene on Metal Surfaces. J. Phys. Chem. C 2012, 116 (13), 7360-7366.

40. Tang, W.; Sanville, E.; Henkelman, G., A grid-based Bader analysis algorithm without lattice bias. J. Phys.: Condens. Matter 2009, 21 (8), 084204.

41. Green, W. H.; Gorun, S. M.; Fitzgerald, G.; Fowler, P. W.; Ceulemans, A.; Titeca, B. C., Electronic Structures and Geometries of $\mathrm{C}_{60}$ Anions via Density Functional Calculations. J. Phys. Chem. 1996, 100 (36), 14892-14898.

42. Vines, F.; Gomes, J. R. B.; Illas, F., Understanding the reactivity of metallic nanoparticles: beyond the extended surface model for catalysis. Chemical Society Reviews 2014.

43. Zöttl, S.; Kaiser, A.; Daxner, M.; Goulart, M.; Mauracher, A.; Probst, M.; Hagelberg, F.; Denifl, S.; Scheier, P.; Echt, O., Ordered phases of ethylene adsorbed on charged fullerenes and their aggregates. Carbon 2014, 69 (0), 206-220. 